Revue d'histoire de l'Amérique française

RAS REVUE D.HISTOIRE DE L'AMÉRIQUE FRANÇAISE

\title{
Faire le saut : la biographie peut-elle être de l'histoire sociale?
}

\section{Suzanne Morton}

Volume 54, numéro 1, été 2000

URI : https://id.erudit.org/iderudit/305660ar

DOI : https://doi.org/10.7202/305660ar

Aller au sommaire du numéro

Éditeur(s)

Institut d'histoire de l'Amérique française

ISSN

0035-2357 (imprimé)

1492-1383 (numérique)

Découvrir la revue

Citer ce document

Morton, S. (2000). Faire le saut : la biographie peut-elle être de l'histoire sociale? Revue d'histoire de l'Amérique française, 54(1), 103-109.

https://doi.org/10.7202/305660ar d'utilisation que vous pouvez consulter en ligne.

https://apropos.erudit.org/fr/usagers/politique-dutilisation/ 


\section{Faire le saut :}

\section{la biographie peut-elle}

être de l'histoire sociale?

SUZANNE MORTON

Département d'histoire

Université McGill

D ANS CETTE SUITE D'ARTICles consacrés à la biographie, je suis incontestablement la non-experte. Je me définis comme spécialiste de l'histoire sociale et, même si j'en comprends les limites sous l'angle épistémologique, j'avoue la considérer, ce qui n'est guère à la mode, comme l'histoire de la société saisie dans toutes ses dimensions, c'est-àdire comme ce que les Annales prétendaient être «l'histoire totale». Les questions historiques qui m'intéressent - celles de communauté, de culture, de valeurs et d'identité, ainsi que leurs rapports aux données de la vie matérielle - mettent en cause, par définition, des groupes d'individus. Cela étant, je me retrouve dans une situation inattendue: celle d'entreprendre une biographie.

Mon adhésion au projet de l'histoire sociale n'est pas étrangère à cette idée selon laquelle l'écriture et l'enseignement de l'histoire constituent un acte politique et moral. Il est essentiel que mes étudiants comprennent que le monde dans lequel ils vivent n'est pas le résultat naturel ou inévitable de la civilisation, mais que des choix ont présidé à la formation du présent. Je veux qu'ils comprennent que le passé est rempli de personnes bien réelles, qui ont vécu une vraie vie et qu'il est possible, même si elles sont disparues à jamais, d'en entrevoir le parcours. Par-dessus tout, je veux qu'ils comprennent que leur vie est définie par l'histoire et qu'ils 
doivent découvrir des manières de saisir la différence entre ce qu'ils ne peuvent pas changer et ce qu'il est en leur pouvoir de changer.

$\mathrm{Si}$ j'évoque ainsi mes étudiants au moment d'expliquer pourquoi j'envisage d'écrire une biographie, c'est qu'ils sont au cœur de mes préoccupations actuelles - ou plutôt qu'ils partagent mes pensées avec une travailleuse sociale nommée Jane Wisdom. La plupart des étudiants que je rencontre sont en première année et suivent le cours d'introduction à l'histoire du Canada depuis 1867. Leur matière principale n'est pas l'histoire et il est plus que probable que ce cours d'histoire sera le seul qu'ils suivront durant tout leur parcours universitaire. Talentueux et provenant de milieux fort divers, ils ne sont ni enthousiastes ni ouverts à la perspective de voir remises en question leurs idées préconçues sur l'histoire canadienne. J'ai découvert, en donnant ce cours, que biographies et autobiographies sont des instruments fort efficaces pour transformer les stéréotypes. Les Attendez que je me rappelle de René Lévesque, Half Breed de Marie Campbell, The Danger Tree: Memory, War and the Search for a Family's Past, de David MacFarlane et The Concubine's Children de Denise Chong, sont tous des ouvrages qui ont exercé un impact profond sur leurs lecteurs.

Cette prise de conscience de l'efficacité et de l'attrait de la biographie et de l'autobiographie recoupe la relation archivistique que j'ai vécue épisodiquement depuis quinze ans avec une obscure travailleuse sociale nommée Jane Wisdom. Durant sa vie, et même après sa mort survenue en 1975, cette femme n'attira guère l'attention. Alors que l'Histoire a retenu le nom de certains de ses collègues et de ses amis, allant jusqu'à leur rendre hommage, l'Université St. Francis Xavier rejetait même la candidature de Jane Wisdom, alors retraitée, à un doctorat honorifique.

Née à Saint-Jean, au Nouveau-Brunswick, en 1884, elle était la deuxième fille d'un homme d'affaires néo-écossais dont les entreprises n'étaient guère florissantes. En 1903, elle suivit sa sœur aînée à l'Université McGill, où elle subit l'influence du mouvement réformiste britannique et se dévoua à l'organisme qui précéda le McGill University Settlement House. Toujours intéressée, après l'obtention de son diplôme, au problème de la pauvreté à Montréal, elle devint une des premières employées rémunérées à titre de "visiteuse", ou d'assistante sociale, de la Montreal Charity Organization Society (COS). En 1910, elle quitta Montréal pour suivre un cours à la New York School of Philanthropy, où elle fut une des premières Canadiennes à recevoir une formation "professionnelle" en travail social; elle fit aussi partie de ces nombreuses 
travailleuses sociales qui firent carrière dans les deux pays. Cette spécialisation terminée, elle revint au COS de Montréal, mais retourna bientôt à New York où on lui proposait, outre la perspective de poursuivre sa formation, un meilleur emploi à titre de secrétaire générale du Brooklyn Bureau of Charities.

En 1916, la ville de Halifax créait son propre service (de coordination) d'aide sociale et retint les services de Wisdom, à cause de sa compétence et de ses nombreux contacts dans le milieu. Seule assistante sociale "professionnelle» à l'époque de l'explosion de 1917, elle fut provisoirement affectée à la Halifax Relief Commission pour y coordonner les interventions auprès des familles au début de la période de reconstruction. Après l'explosion, des travailleurs sociaux de partout en Amérique du Nord (mais surtout de Montréal) constituèrent un volet important des opérations de secours à la fois modernes, rationnelles et bureaucratiques. L'état d'urgence étant passé, Wisdom reprit en 1919 son poste au Halifax Welfare Bureau. Directrice du plus important organisme non confessionnel d'aide sociale de la Nouvelle-Écosse, elle fut appelée, en 1920, à participer à une enquête gouvernementale sur les pensions de retraite et sur le salaire minimum accordé aux femmes, devenant ainsi la première femme membre d'une commission royale d'enquête dans cette province. En 1921, elle démissionna de son poste au Halifax Welfare Bureau et retourna à Montréal afin d'y acquérir, à l'Université McGill, un diplôme en histoire et en économie. Lors des grandes agitations ouvrières de 1922, elle interrompit sa thèse, qui portait sur les conditions sociales des communautés minières du Cap Breton, et gagna la Nouvelle-Écosse où elle rédigea, durant cette période de crise, une chronique quotidienne dans le Sydney Post. Revenue à Montréal, elle n'y poursuivit pas ses études, mais agit plutôt comme secrétaire générale du Montreal's Women Directorate, organisme protestant consacré à l'aide aux mères célibataires. Elle allait conserver ce poste jusqu'en 1939, travaillant en outre à la Montreal School of Social Work, au Montreal Council of Social Agencies, à diverses organisations professionnelles et à un réseau officieux de travailleuses sociales regroupées autour de Charlotte Whitton. Après sa retraite, en 1939, Wisdom fut choisie pour enquêter sur les services sociaux de Glace Bay, au Cap Breton, sous les auspices du Canadian Council on Child and Family Welfare et du District 26 des United Mine Workers of America. Elle recommanda, dans son rapport, que la ville de Glace Bay fasse entorse aux lois provinciales sur l'assistance publique, jugées trop 
restrictives, et substitue au "surveillant des pauvres " un travailleur social municipal. Non seulement cette audacieuse réforme fut-elle acceptée, mais on persuada Wisdom d'occuper elle-même cette fonction, ce qu'elle fit jusqu'en 1954.

Comme historienne, le nom de Jane Wisdom m'est apparu pour la première fois alors que je menais une recherche sur la grève de $1922 \mathrm{au}$ Cap Breton. J'allais ensuite le retrouver en étudiant la Commission royale d'enquête évoquée précédemment et les archives relatives à l'explosion et à la reconstruction de Halifax. Ce nom allait de nouveau refaire surface dans la documentation sur les mères célibataires durant l'entre-deux-guerres et sur les réformes apportées aux lois de l'assistance publique en Nouvelle-Écosse après la Deuxième Guerre mondiale. Déménagée entre-temps à Montréal, j'en vins à diriger des travaux et des mémoires de maitrise sur les institutions d'aide sociale à Montréal. J'ai souvent l'impression que non seulement Jane Wisdom avait le don de surgir en des lieux passionnants, mais que mon travail m'amène à suivre sa carrière à la trace.

J'en suis venue à croire que cette vie nous ouvre une fenêtre sur ce que fut le travail social durant la première moitié du $\mathrm{xx}^{\mathrm{e}}$ siècle. En suivant cette carrière, je suis à même d'examiner l'histoire des politiques sociales et celle de l'instauration de l'État-providence par le biais des centres d'œuvres sociales, de l'action des philanthropes, du progressisme bureaucratique, des commissions gouvernementales et de l'activisme syndical. Son cheminement professionnel renvoie aux débats sur le droit aux prestations d'aide sociale, sur les questions de genre et sur les mesures adoptées localement durant la crise économique des années 1930. En outre, le fait que sa carrière se soit déroulée dans des lieux où prévalaient des systèmes d'aide sociale différents - New York, Montréal et des villes de la Nouvelle-Écosse - autorise diverses approches comparatives. La biographie m'apparaît donc comme riche de possibilités pour la microhistoire ou l'étude de cas. Je suis particulièrement inspirée par l'idée d'une "histoire sociale», évoquée par David Frank dans sa biographie du leader syndical J. B. McLachlan. Dans cet ouvrage, Frank rappelle au lecteur le propos de Nick Salvatore, lui-même biographe de Eugene V. Debs: si la biographie s'attache à un individu, "elle est aussi un fragment d'histoire sociale dans la mesure où l'individu n'existe pas hors de la culture et de la société dans lesquelles il a grandi et dont il émane ${ }^{1}$ ».

1. Nick Salvatore, Eugene V. Debs: Citizen and Socialist (Urbana, III, 1982), xi ; David Frank, J. B. McLachlan: A Biography (Toronto, 1999). 
J'aborde aussi la biographie à partir des récents travaux féministes dans ce domaine. Il n'est pas sans signification, selon moi, que Jane Wisdom ait été une femme, qu'à l'instar de la plupart des femmes elle n'ait pas été considérée comme "importante» et que, jusqu'à tout récemment, le genre biographique ait été d'esprit conservateur et dominé par des hommes qui narraient la vie d'autres hommes «importants». L'étude de la vie d'une seule femme nous permet de voir à l'œuvre le pouvoir patriarcal qui a dessiné le monde de Jane Wisdom et d'entrevoir comment cette dernière a tiré profit de sa classe sociale et de son appartenance ethnique pour reproduire ce pouvoir.

Cette ambiguiité d'une Wisdom, à la fois victime et bienfaitrice, ajoute à l'intérêt d'une analyse centrée sur la vie d'un individu. La biographe Meryn Stuart écrit avec justesse: «Bien des choses n'acquièrent de sens qu'illustrées par la vie d'une seule personne ${ }^{2}$.» Une vie individuelle permet une ligne narrative claire. En recourant au schéma classique - de la naissance à la mort - il est possible d'explorer les données particulières d'une vie, tout en évoquant ses composantes normatives. Je pense ici à des biographies politiques à la fois importantes et faciles d'accès, comme George-Étienne Cartier: Montreal Bourgeois, de Brian Young (1981) et The Life of Lester Pearson: Shadow of Heaven, 1897-1948 et Worldly Years, 1949-1972, de John English (1989 et 1992). Si ces ouvrages s'enracinent dans l'histoire politique, certains des plus influents travaux d'histoire sociale des trente dernières annnées peuvent être considérés comme des formes de biographie qui prennent en compte le social. Ainsi, les ouvrages suivants partagent certaines catactéristiques de la biographie collective: Le fromage et les vers: l'univers d'un meunier $d u \quad x V I^{e}$ siècle, de Carlo Ginzburg (1980) (orig.: Il formaggio e i vermi), The Return of Martin Guerre et Women on the margins: Three Seventeenth-Century Lives de Natalie Zemon Davis (1983 et 1995) et même Family Fortunes: Men and Women of the English Middle Class, 1780-1850, une étude centrée sur deux familles par Leonore Davidoff et Catherine Hall (1987).

Mais entreprendre la biographie d'une obscure assistante sociale ne va pas sans problèmes sérieux dont, au premier chef, le fait que ses documents personnels ont vraisemblablement été perdus après son décès. Si l'on en croit certaines rumeurs, la famille les aurait prêtés à un étudiant désireux d'en faire le matériel de base d'un mémoire de maîtrise et qui ne les aurait jamais rendus après avoir abandonné son projet. Deux

2. Meryn Stuart, "Making the Choices: Writing About Marguerite Carr-Harris», in Teresa Iles, All Sides of the Subject: Women and Biography (New York, 1992), 59. 
raisons me portent à ajouter foi à cette histoire : d'une part, quelqu'un a consulté ces documents en 1977 et, d'autre part, Wisdom appartenait à une famille qui avait l'habitude de conserver ses propres documents et même de les déposer aux archives publiques. Sa nièce, Relief Williams McKay, fut une des premières femmes à obtenir, en 1936, une maitrise en histoire à l'Université McGill. Elle présida même au transfert d'autres documents de famille aux archives de la Nouvelle-Écosse, de sorte que je me trouverai, situation bizarre, à avoir accès à la correspondance de la mère et de la tante de Jane, mais non à la sienne. Mais si ses documents personnels sont perdus à jamais (à l'importante exception de ses travaux d'étudiante qui aboutirent aux archives de McGill), la plupart des organisations pour lesquelles elle travailla ont confié leurs dossiers aux archives publiques. Il se peut donc que la vie privée de Jane Wisdom échappe pour toujours au biographe, mais sa carrière et une grande partie de sa correspondance professionnelle ont traversé les temps.

Je suis encore habitée par l'importante question qui me hantait quant à la manière d'aborder une biographie. La carrière d'une simple travailleuse sociale, à laquelle j'espère rattacher l'histoire de l'État-providence, suffirait-elle à retenir l'intérêt du lecteur? Certaines raisons me laissent croire que la réponse est positive. Divers éléments historiques militent en ce sens: l'état dramatique des taudis du Montréal d'avant la Première Guerre mondiale, la communauté des immigrants de New York, l'explosion de Halifax, les grèves des années 1920 au Cap Breton et le Montréal de l'entre-deux-guerres. Un récit articulé autour de la vie de Jane Wisdom illustrerait les tendances dominantes dans les domaines du travail social, des attitudes de la population et d'un État-providence en émergence. De plus, l'ouvrage dépeindrait le travailleur social et le réformateur social en tant qu'individu. Au cours des derniers vingt ans, les historiens se sont efforcés de retracer et d'inclure dans leurs travaux la voix des marginaux et des opprimés. L'histoire n'en est que plus riche, certes, mais ces préoccupations ont eu pour conséquence involontaire de créer l'image unidimensionnelle du réformateur attaché à régenter, à condamner et désapprouver ceux-là mêmes qu'il entendait aider. Appréhendée dans son individualité, Jane Wisdom est beaucoup plus complexe que cela. Formée par sa culture et par son statut social mais engagée durant toute sa vie à améliorer la situation sociale, elle fut à la fois conformiste et déviante. Elle n'atteignit sans doute pas, à son époque, à la célébrité, mais son action a fait évoluer les choses. Et cela signifie 
peut-être, à une époque et dans une culture qui doute désormais de la véritable influence des individus, qu'une biographie sociale peut comporter un fort utile message politique et moral.

Traduction: Pierre R. Desrosiers 